\title{
Genomic surveillance of Pseudomonas aeruginosa in the Philippines, 2013-2014
}

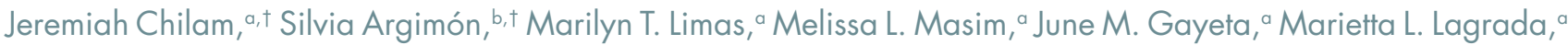
Agnettah M. Olorosa, ${ }^{a}$ Victoria Cohen, ${ }^{b}$ Lara T. Hernandez, ${ }^{a}$ Benjamin Jeffrey, ${ }^{b}$ Khalil Abudahab, ${ }^{b}$ Charmian M. Hufano, ${ }^{a}$ Sonia B. Sia, ${ }^{a}$ Matthew T.G. Holden, ${ }^{d}$ John Stelling, ${ }^{c}$ David M. Aanensen, ${ }^{b, e,},{ }^{\star}$ and Celia C. Carlos, ${ }^{a,{ }^{*}}$ on behalf of the Philippines Antimicrobial Resistance Surveillance Program

Correspondence to Celia Carlos (email: ccarlosphl@gmail.com) and David M. Aanensen (email: David.aanensen@bdi.ox.ac.uk)

Pseudomonas aeruginosa is an opportunistic pathogen that often causes nosocomial infections resistant to treatment. Rates of antimicrobial resistance (AMR) are increasing, as are rates of multidrug-resistant (MDR) and possible extensively drug-resistant (XDR) infections. Our objective was to characterize the molecular epidemiology and AMR mechanisms of this pathogen.

We sequenced the whole genome for each of 176 P. aeruginosa isolates collected in the Philippines in 2013-2014; derived the multilocus sequence type (MLST), presence of AMR determinants and relatedness between isolates; and determined concordance between phenotypic and genotypic resistance.

Carbapenem resistance was associated with loss of function of the OprD porin and acquisition of the metallo- $\beta$-lactamase $(\mathrm{MBL})$ gene blavim. Concordance between phenotypic and genotypic resistance was $93.27 \%$ overall for six antibiotics in three classes, but varied among aminoglycosides. The population of $P$. aeruginosa was diverse, with clonal expansions of XDR genomes belonging to MLSTs ST235, ST244, ST309 and ST773. We found evidence of persistence or reintroduction of the predominant clone ST235 in one hospital, and of transfer between hospitals.

Most of the ST235 genomes formed a distinct lineage from global genomes, thus raising the possibility that they may be unique to the Philippines. In addition, long-read sequencing of one representative XDR ST235 isolate identified an integron carrying multiple resistance genes (including b/a VIM-2 $_{2}$ ), with differences in gene composition and synteny from the $P$. aeruginosa class 1 integrons described previously.

The survey bridges the gap in genomic data from the Western Pacific Region and will be useful for ongoing surveillance; it also highlights the importance of curtailing the spread of ST235 within the Philippines.

$\mathrm{P}$ seudomonas aeruginosa is an opportunistic pathogen that often causes nosocomial infections (e.g. pneumonia, bacteraemia and urinary tract infections), particularly in immunocompromised patients. ${ }^{1}$ Eight Asian countries reported frequencies of isolation of Pseudomonas spp. of above $15 \%$ from hospital-acquired (HA) pneumonia cases, with the Philippines reporting $P$. aeruginosa as the most common etiological agent. $^{2}$ Also, Pseudomonas spp. were the second most common pathogen isolated from deviceassociated $\mathrm{HA}$ infections in a study of intensive care units in Philippine hospitals. ${ }^{3}$
$P$. aeruginosa infections are often resistant to treatment, ${ }^{4}$ and carbapenem use has been strongly associated with resistance. ${ }^{1}$ However, a study evaluating carbapenem restriction practices at a hospital in Manila found that $37 \%$ of the carbapenem prescriptions were non-compliant, highlighting challenges in antimicrobial stewardship. ${ }^{5}$ Between 2010 and 2014, the Philippine Antimicrobial Resistance Surveillance Program (ARSP) reported increasing rates of resistance to antibiotics used to treat $P$. aeruginosa infections, such as carbapenems and extended-spectrum cephalosporins (Fig. 1A-B). In contrast, resistance to aminoglycosides and fluoroqui-

\footnotetext{
Antimicrobial Resistance Surveillance Reference Laboratory, Research Institute for Tropical Medicine, Muntinlupa, Philippines.

Centre for Genomic Pathogen Surveillance, Wellcome Genome Campus, Hinxton, England, United Kingdom of Great Britain and Northern Ireland. Brigham and Women's Hospital, Boston (MA), USA.

University of St Andrews School of Medicine, St Andrews, Scotland, United Kingdom of Great Britain and Northern Ireland.

Centre for Genomic Pathogen Surveillance, Big Data Institute, University of Oxford, Oxford, England, United Kingdom of Great Britain and Northern Ireland.

These authors contributed equally to this work.

These authors contributed equally to this work.

Published: 28 April 2021

doi: 10.5365/wpsar.2020.11.1.006
} 
Fig. 1A-C. Annual resistance rates to nine antibiotics of $P$. aeruginosa isolates referred to the ARSP, 2005-2014
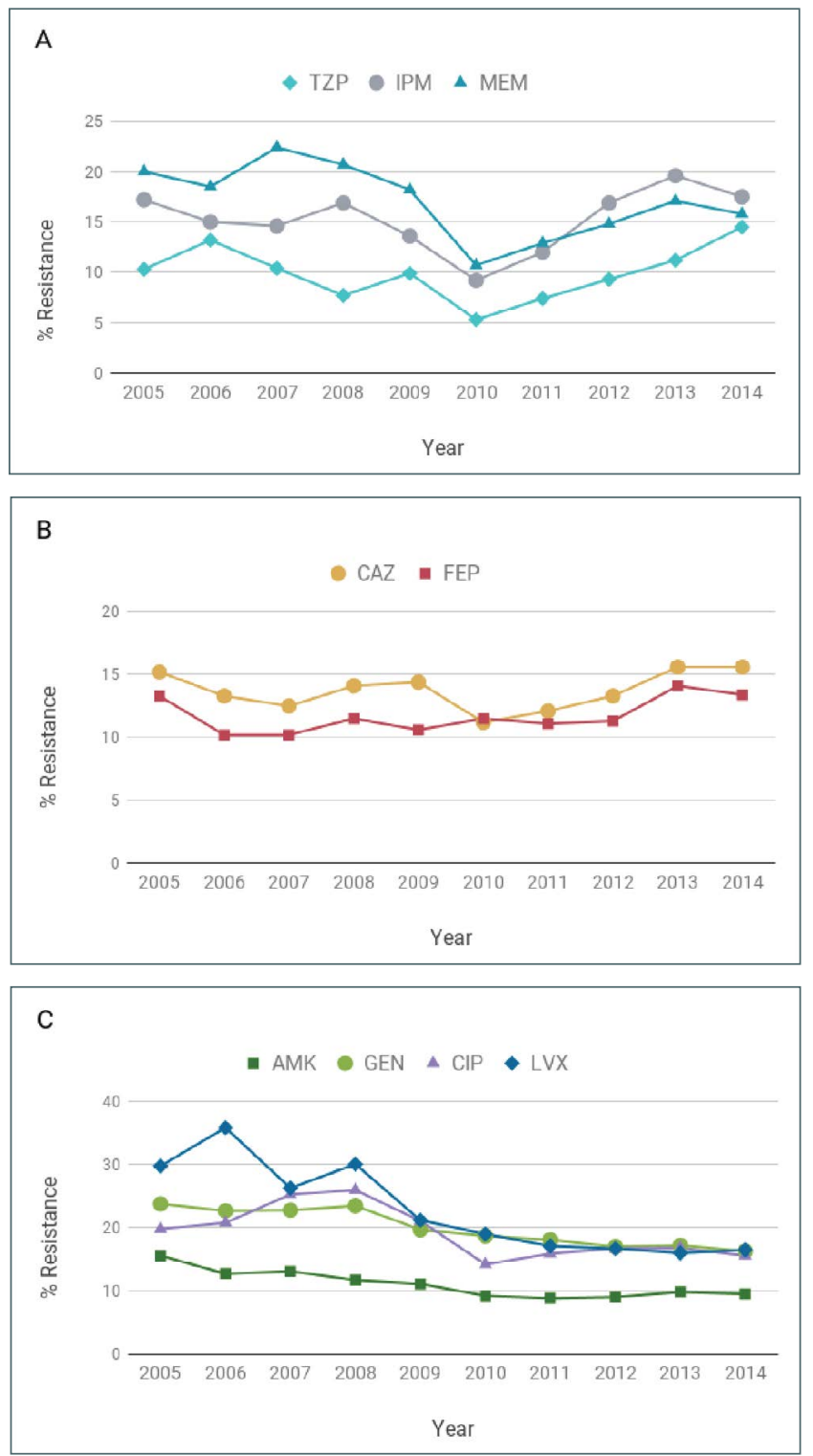

AMK: amikacin; CAZ: ceftazidime; CIP: ciprofloxacin; FEP: cefepime; GEN: gentamicin; IPM: imipenem; LVX: levofloxacin; MEM: meropenem, TZP: piperacillin-tazobactam.

nolones remained relatively stable or decreased slightly in the same period (Fig. 1C). The ARSP has also reported multidrug-resistant (MDR) rates of $21-23 \%$ and possible extensively drug-resistant (XDR) rates of $13-18 \%$ in recent years. ${ }^{6-8}$

The emergence of MDR $P$. aeruginosa with resistance to carbapenems, aminoglycosides and fluoroquinolones was followed by reports of isolates sensitive only to colistin ${ }^{9}$ and, more recently, of colistin resistance in carbapenem non-susceptible isolates, ${ }^{10}$ leaving few treat- ment options. These reports coincide with multi-locus sequence type (MLST) ST235, ${ }^{9-11}$ the predominant global epidemic clone. The metallo- $\beta$-lactamase (MBL) genes blavim and bla $a_{\text {IMP }}$ - usually associated with integrons carrying multiple resistance determinants - have been identified in ST235 $P$. aeruginosa isolates from Asian countries. ${ }^{12-14}$

While the resistance rates and profiles of $P$. aeruginosa in the Philippines have been well characterized, ${ }^{15,16}$ the molecular epidemiology and AMR mechanisms of this pathogen remain largely unknown. Whole-genome sequencing (WGS) can identify transmission patterns, AMR mechanisms and the source of HA infections. ${ }^{17}$ In this study, we characterized the clonal relatedness and resistance determinants of $P$. aeruginosa isolates from the ARSP using WGS.

\section{METHODS}

\section{Bacterial isolates}

A total of $7877 P$. aeruginosa isolates were collected and tested for resistance by the ARSP from January 2013 to December 2014. Of the 443 and 283 isolates referred to the Antimicrobial Resistance Surveillance Reference Laboratory (ARSRL) for confirmation in 2013 and 2014, respectively, 179 isolates from 17 sentinel sites were selected for WGS, as previously described. ${ }^{18}$ Briefly, 113 isolates of carbapenemase-producing $P$. aeruginosa were selected; also included were 66 available isolates that were susceptible to all antibiotics tested. We used a proxy definition for "infection origin", whereby initial infection isolates collected in the community or on either of the first 2 days of hospitalization were categorized as communityacquired (CA), and isolates collected on hospital day 3 or later were categorized as hospital-acquired $(\mathrm{HA}){ }^{19}$

\section{Antimicrobial susceptibility testing (AST)}

All $P$. aeruginosa isolates from this study were tested for susceptibility to nine antibiotics representing five classes: amikacin (AMK), ceftazidime (CAZ), ciprofloxacin (CIP), cefepime (FEP), gentamicin (GEN), imipenem (IPM), meropenem (MEM), tobramycin (TOB), and piperacillintazobactam (TZP) (Table 1). Antimicrobial susceptibility of the isolates was determined at ARSRL using the KirbyBauer disk diffusion method, and gradient methods such as E-Test (bioMérieux, Marcy-l'Étoile, France) and Vitek 2 
Table 1. Total number of $\boldsymbol{P}$. aeruginosa isolates analysed by the ARSP and referred to the ARSRL during 2013 and 2014, isolates submitted for WGS, and high-quality P. aeruginosa genomes obtained, discriminated by sentinel site and AMR profile

\begin{tabular}{|c|c|c|c|}
\hline & \multicolumn{3}{|c|}{ Number of isolates } \\
\hline & 2013 & 2014 & Total \\
\hline Total ARSP & 3591 & 4286 & 7877 \\
\hline Referred to ARSRL & 443 & 283 & 726 \\
\hline Submitted for WGS & 89 & 90 & 179 \\
\hline High-quality genomes & 87 & 89 & 176 \\
\hline \multicolumn{4}{|l|}{ By sentinel site * } \\
\hline $\mathrm{BGH}$ & 2 & 4 & 6 \\
\hline $\mathrm{BRH}$ & 0 & 5 & 5 \\
\hline CMC & 0 & 1 & 1 \\
\hline CVM & 2 & 3 & 5 \\
\hline DMC & 5 & 2 & 7 \\
\hline EVR & 2 & 2 & 4 \\
\hline FEU & 2 & 2 & 4 \\
\hline GMH & 4 & 4 & 8 \\
\hline JLM & 2 & 5 & 7 \\
\hline $\mathrm{MMH}$ & 3 & 5 & 8 \\
\hline NKI & 10 & 16 & 26 \\
\hline NMC & 3 & 8 & 11 \\
\hline RMC & 2 & 0 & 2 \\
\hline SLH & 0 & 1 & 1 \\
\hline STU & 5 & 4 & 9 \\
\hline VSM & 32 & 16 & 48 \\
\hline \multicolumn{4}{|l|}{ By AMR profile ** } \\
\hline Susceptible & 36 & 30 & 66 \\
\hline CAZ FEP IPM MEM TZP GEN TOB AMK CIP [XDR] & 30 & 29 & 59 \\
\hline IPM MEM & 7 & 9 & 16 \\
\hline CAZ FEP IPM MEM TZP GEN TOB CIP [XDR] & 4 & 7 & 11 \\
\hline CAZ FEP IPM MEM TZP GEN TOB AMK & 1 & 4 & 5 \\
\hline CIP & 3 & 2 & 5 \\
\hline CAZ FEP IPM MEM TZP & 1 & 2 & 3 \\
\hline IPM MEM TZP CIP & 0 & 1 & 1 \\
\hline GEN TOB CIP & 1 & 0 & 1 \\
\hline FEP TZP TOB CIP & 0 & 1 & 1 \\
\hline CAZ FEP IPM MEM GEN TOB & 1 & 0 & 1 \\
\hline IPM & 1 & 0 & 1 \\
\hline CAZ FEP IPM MEM GEN TOB CIP & 1 & 0 & 1 \\
\hline IPM MEM CIP & 1 & 0 & 1 \\
\hline CAZ FEP GEN TOB AMK CIP & 0 & 1 & 1 \\
\hline FEP IPM MEM GEN TOB CIP & 0 & 1 & 1 \\
\hline $\mathrm{CAZ}$ & 0 & 1 & 1 \\
\hline CAZ FEP TZP & 0 & 1 & 1 \\
\hline
\end{tabular}

* AMK: amikacin; AMR: antimicrobial resistance; ARSP: Antimicrobial Resistance Surveillance Program; ARSRL: Antimicrobial Resistance Surveillance Reference Laboratory; CAZ: ceftazidime; CIP: ciprofloxacin; FEP: cefepime; GEN: gentamicin; IPM: imipenem; MEM: meropenem; TOB: tobramycin; TZP: piperacillin-tazobactam; XDR: extensively drug resistant; WGS: whole-genome sequencing.

** BGH: Baguio General Hospital and Medical Center; BRH: Batangas Medical Center; CMC: Cotabato Regional and Medical Center; CVM: Cagayan Valley Medical Center; DMC: Southern Philippines Medical Center; EVR: Eastern Visayas Regional Medical Center; FEU: Far Eastern University Nicanor Reyes Medical Foundation; GMH: Governor Celestino Gallares Memorial Hospital; JLM: Jose B. Lingad Memorial Regional Hospital; MMH: Corazon Locsin Montelibano Memorial Regional Hospital; NKI: National Kidney and Transplant Institute; NMC: Northern Mindanao Medical Center; RMC: Rizal Medical Center; SLH: San Lazaro Hospital; STU: University of Santo Tomas Hospital; VSM: Vicente Sotto Memorial Medical Center. 
Compact automated system (bioMérieux). To determine the resistance profile of the isolates, the zone of inhibition and minimum inhibitory concentration of antibiotics were interpreted according to guidelines from the Clinical and Laboratory Standard Institute (CLSI). ${ }^{20}$ MDR phenotypes were classified according to standard definitions. ${ }^{21}$

\section{DNA extraction and WGS}

A total of $179 P$. aeruginosa isolates were shipped to the Wellcome Trust Sanger Institute for WGS. DNA was extracted from a single colony of each isolate with the QIAamp 96 DNA QIAcube HT kit and a QIAcube HT (Qiagen, Hilden, Germany). DNA extracts were multiplexed and sequenced on the Illumina HiSeq platform (Illumina, CA, USA) with 100-bp paired-end reads. Isolate 13ARSVSM740 was also sequenced with the PacBio RSII platform (Pacific Biosciences). Raw sequence data were deposited in the European Nucleotide Archive (ENA) under the study accession PRJEB17615. Run accessions for Illumina data are provided on the Microreact projects. The PacBio data were deposited under run accession ERR3284501.

\section{Bioinformatics analysis}

Genome quality was evaluated based on metrics generated from assemblies, annotation files and the alignment of the isolates to the reference genome of $P$. aeruginosa strain LESB58 (accession FM209186), as previously described. ${ }^{18}$ Assemblies were produced from short-read Illumina data ${ }^{18}$ and from long-read PacBio data with the HGAP v4 pipeline (Pacific Biosciences). A total of 176 isolates yielded high-quality $P$. aeruginosa genomes and were included in this study.

We derived the MLST of the isolates from the whole genome sequences. The sequence types (ST) were determined from assemblies with Pathogenwatch (https:// pathogen.watch/) and with MLSTcheck v1.007001, and from sequence reads with $\mathrm{ARIBA}^{22}$ and the $P$. aeruginosa database hosted at PubMLST. ${ }^{23}$ The MLST calls were curated, as previously described..$^{18}$ Integrons were detected in the genome assemblies with IntegronFinder. ${ }^{24}$

Evolutionary relationships between the 176 isolates were inferred from core single-nucleotide polymorphism (SNP). A core gene alignment was performed with Roary v3.11.3, using the mafft aligner option and minimum percentage identity for blastp of $90 \%$. Evolutionary relationships between 169 isolates from groups 1 and 2 were inferred from SNPs by mapping the paired-end reads to the reference genomes of $P$. aeruginosa strains LESB58 (ST146, FM209186) or NCGM2_S1 (ST235, AP012280.1). ${ }^{18}$ Mobile genetic elements (MGEs) were masked in the alignment of pseudogenomes with a script available at https://github.com/sanger-pathogens/ remove_blocks_from_aln. For the phylogenetic analysis of ST235 genomes, recombination regions detected with Gubbins ${ }^{25}$ were also removed. Alignments of SNPs were inferred with snp-sites v2.4.1, ${ }^{26}$ and were used to compute pairwise SNP differences between isolates from different patients (minimum $n=3$ ) belonging to the same or to different hospitals, using a script from https:// github.com/simonrharris/pairwise_difference_count. Maximum likelihood phylogenetic trees were generated with RAxML, ${ }^{27}$ based on the generalized time reversible (GTR) model with GAMMA method of correction for among-site rate variation and 100 bootstrap replications.

To contextualize the Philippine genomes, we downloaded, assembled and quality controlled global $P$. aeruginosa genomes with linked geographical and temporal information, collected mainly between 2007 and 2017, for which raw Illumina paired-end sequence data were available at the ENA. A tree of 904 genomes was inferred with FastTree ${ }^{28}$ from an alignment of 549126 SNP positions, obtained after mapping the reads to the complete genome of strain LESB58 and masking regions with MGEs. A tree of 96 global ST235 genomes was inferred with RAxML from an alignment of 1993 SNP sites obtained after mapping the genomes to the complete genome of strain NCGM2-S1, and masking MGEs and recombination regions.

Known AMR determinants were identified with ARIBA $^{22}$ and a curated database of known resistance genes and mutations, ${ }^{29}$ the Comprehensive Antibiotic Resistance Database, ${ }^{30}$ and a custom database of mutations in the quinolone resistance-determining region of the gyrA/B and parC/E genes described for $P$. aeruginosa. ${ }^{4}$ The output for the porin gene oprD was inspected to detect loss-of-function mutations. The oprD sequences were extracted from the whole-genome draft assemblies with blastn, using the oprD sequence from strain PAO1 (accession NC_002516.2, genome positions 1043982-1045314) as a query, then translated in silico to inspect the integrity of the coding frames. A 444 or 
442 amino-acid protein that included a START and a STOP codon was considered functional.

The genomic predictions of AMR derived from the presence of known AMR genes and mutations (test) were compared with the phenotypic results (reference), and concordance was computed for each of six antibiotics (1056 total comparisons). Isolates with either a resistant or an intermediate phenotype were considered non-susceptible. An isolate with the same outcome for both the test and reference (i.e. both susceptible or both non-susceptible) was counted as a concordant isolate. Concordance was the number of concordant isolates as a percentage of the total number of isolates assessed.

All project data, including inferred phylogenies, AMR predictions and metadata were made available through Microreact.

\section{Ethics statement}

Ethical approval is not applicable. This study uses archived bacterial samples processed by the ARSP. No identifiable data were used in this study.

\section{RESULTS}

\section{Demographic and clinical characteristics of the $P$. aeruginosa isolates}

Of the $179 P$. aeruginosa isolates submitted for WGS, 176 passed quality control and were confirmed in silico as $P$. aeruginosa (Table 2). Patients were aged from under 1 to 96 years, with $27 \%(n=47)$ of the isolates from patients aged 65 years or older. Fifty-eight per cent $(n=102)$ of the isolates were from HA infections. In terms of specimen type, $53 \%(n=94)$ of isolates were from respiratory samples (tracheal aspirates and sputum).

\section{Concordance between phenotypic and geno- typic AMR}

Isolates were tested for susceptibility to nine antibiotics representing five classes (Fig. 1A-C, Table 3). Most isolates were non-susceptible to carbapenems ( $n=$ 100), 10 isolates were susceptible to carbapenems but resistant to other antibiotics, and 66 isolates were susceptible to all nine antibiotics (Table 1). CA infections
Table 2. Demographic and clinical characteristics of $176 P$. aeruginosa isolates ${ }^{a}$

\begin{tabular}{|c|c|}
\hline Characteristic & No. isolates \\
\hline \multicolumn{2}{|l|}{ Sex } \\
\hline Male & 119 \\
\hline Female & 57 \\
\hline \multicolumn{2}{|l|}{ Age (in years) } \\
\hline$<1$ & 12 \\
\hline $1-4$ & 6 \\
\hline $5-14$ & 7 \\
\hline $15-24$ & 14 \\
\hline $25-34$ & 5 \\
\hline $35-44$ & 17 \\
\hline $45-54$ & 29 \\
\hline $55-64$ & 34 \\
\hline $65-80$ & 36 \\
\hline$\geq 81$ & 11 \\
\hline Age unknown & 5 \\
\hline \multicolumn{2}{|l|}{ Patient type } \\
\hline Inpatient & 159 \\
\hline Outpatient & 17 \\
\hline \multicolumn{2}{|l|}{ Specimen origin } \\
\hline Community-acquired & 74 \\
\hline Hospital-acquired & 102 \\
\hline \multicolumn{2}{|l|}{ Submitted as } \\
\hline Carbapenem non-susceptible & 100 \\
\hline $\begin{array}{l}\text { Resistant to at least } 1 \text { antibiotic other than } \\
\text { carbapenems }\end{array}$ & 10 \\
\hline Susceptible & 66 \\
\hline \multicolumn{2}{|l|}{ Specimen type } \\
\hline Abdominal fluid* & 1 \\
\hline Abscess & 1 \\
\hline Blood* & 21 \\
\hline Bronchial & 1 \\
\hline Catheter & 2 \\
\hline Cerebrospinal fluid* & 3 \\
\hline Cornea & 2 \\
\hline Dialysis fluid* & 1 \\
\hline Drainage & 1 \\
\hline Fluid & 3 \\
\hline Inguinal & 1 \\
\hline Other & 1 \\
\hline Pleural fluid* & 1 \\
\hline Sputum & 31 \\
\hline Tissue & 5 \\
\hline Tracheal & 1 \\
\hline Tracheal aspirate & 63 \\
\hline Urine & 12 \\
\hline Wound & 25 \\
\hline
\end{tabular}

a Invasive isolates were considered as those obtained from specimen types marked with an asterisk $(*)$ 
Fig. 1D. Association between resistance and the origin of infection for $176 P$. aeruginosa isolates sequenced in this study

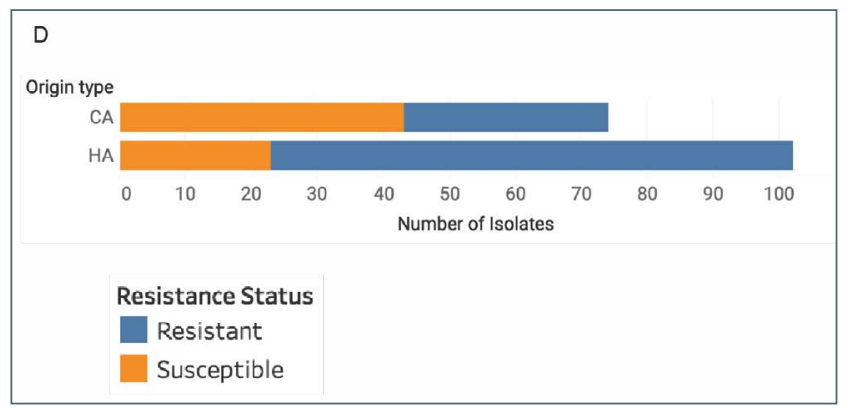

CA: community-acquired; HA: hospital-acquired; Resistant: Resistant to at least one antibiotic tested; Susceptible: Susceptible to all nine antibiotics tested.

were more frequently associated with susceptible isolates and HA infections with resistant isolates (Fig. 1D, twotailed Fisher's exact test $P=0.000002$ ).

Of the 18 isolates resistant to imipenem and meropenem but not to other $\beta$-lactam antibiotics, 17 carried both loss-of-function disruptions in the OprD porin, and disruptions or known non-synonymous mutations in the NalC (A186T, G71E, S209R) and/or NaID (S32N) regula- tors of the MexAB-OprM multidrug efflux pump, suggesting that their resistance is due to a combination of reduced influx and increased efflux of the carbapenem antibiotics (Fig. 1E). Among the 81 carbapenem-resistant isolates that were also resistant to third-generation cephalosporin ceftazidime and/or fourth-generation cephalosporin cefepime, 67 isolates carried acquired MBL genes b/a $a_{\text {VIM-2 }}$ ( $n=61$ genomes), blaviM-6 $(n=1)$, bla IMP-26 $(n=4)$ or bla NDM-1 $_{1}(n=1)$, while five carried disrupted oprD genes plus acquired extended-spectrum $\beta$-lactamase (ESBL) genes bla PER-1 $(n=3)$, bla $a_{\text {CTX-M-15 }}(n=1)$ or AmpC-like gene bla $a_{\mathrm{DHA}-1}(n=1)$. The remaining eight isolates harboured other $\beta$-lactamase genes, but their carbapenemresistance mechanisms remain uncharacterized. Of the 76 isolates susceptible to carbapenems, 75 carried either a full-length OprD porin (444 amino acids) without any known mutations, or a 442 amino acid-long OprD protein with an intact reading frame, while one isolate was missing the STOP codon in the oprD gene.

The overall phenotypic and genotypic concordance was $93.27 \%$ for the six antibiotics analysed (Table 3 ). The concordance was above $96 \%$ for carbapenems.

Fig. 1E. Mechanisms of resistance to carbapenems and other $\beta$-lactam antibiotics identified in the genomes of 176 isolates grouped by their resistance profile

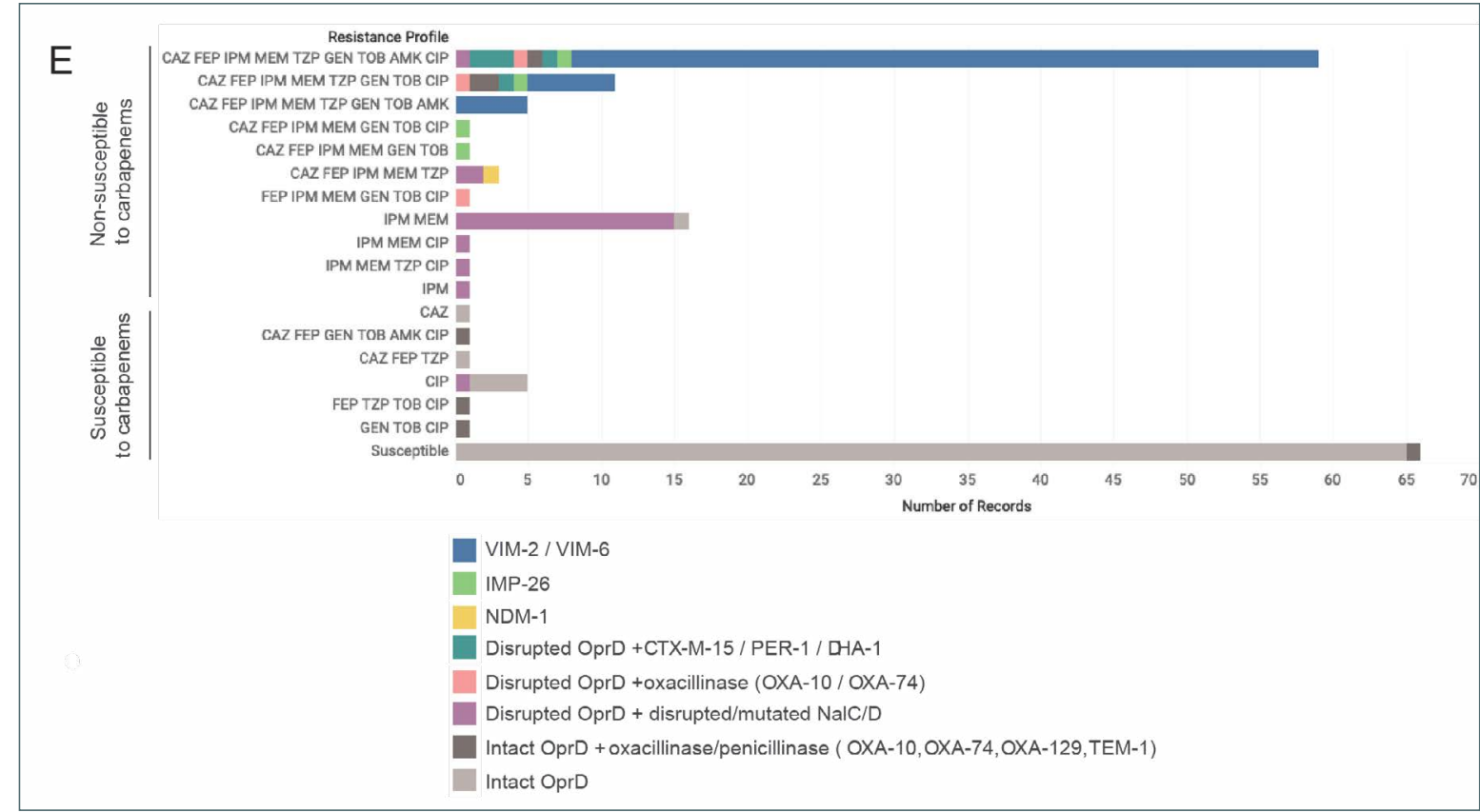

a For simplicity, only the main mechanism is indicated

AMK: amikacin; CAZ: ceftazidime; CIP: ciprofloxacin; FEP: cefepime; GEN: gentamicin; IPM: imipenem; MEM: meropenem; TOB: tobramycin; TZP: piperacillin-tazobactam. 


\section{Genotypic findings}

\section{In silico genotyping}

A total of 79 STs were identified (Table 4), with 27.8\% ( $n=49$ ) belonging to ST235, followed by ST309 $(5.7 \%, n=10)$, ST244 and ST773 (5.1\% each, $n=9)$. The majority of the STs $(79.7 \%, n=63)$ were singletons (represented by only one genome), most of which $(n=42)$ were contributed by the susceptible isolates. Indeed, the resistant isolates (36 STs, $n=110$ ) exhibited less clonal diversity than the susceptible isolates (56 STs, $n=66$ ). ST235 represented $43.6 \%(n=48)$ of the resistant isolates but only $1.5 \%$ $(n=1)$ of the susceptible isolates, and was predominantly a nosocomial clone in the Philippines (36 HA vs $13 \mathrm{CA}$ isolates), spread across 13 hospitals.

\section{Population structure of $P$. aeruginosa in the Philip- pines}

The phylogenetic tree of 176 genomes from the Philippines comprises three major groups, ${ }^{31}$ group $1(n=$ 64) including PA14, group 2 ( $n=105)$ including PAO1 and the more distantly related group $3(n=7)$ including PA7 (Fig. 2A). All three groups included carbapenemresistant isolates and susceptible isolates, though most isolates in group 2 were susceptible $(n=39,60.9 \%)$ and most in group 1 were resistant $(n=75,71.4 \%$, Fig. 2B).

The population of $P$. aeruginosa comprises a limited number of widespread clones selected from a diverse pool of rare, unrelated genotypes that recombine at high frequency. ${ }^{32}$ A phylogenetic tree of 169 genomes from groups 1 and 2 showed that the clonal expansions were mostly within the major group 1 - represented by ST235, ST309, ST773 and ST313 (Fig. 2B) - found across multiple hospitals and resistant to multiple antibiotics. Most of the XDR isolates ( $n=61,87 \%$ ) were found in ST235, ST244, ST309 and ST773, and most ( $n=44,62.8 \%$ ) carried blavIM (an MBL that can degrade all anti-pseudomonal $\beta$-lactamases except for aztreonam), ${ }^{1}$ AAC(6')-Ib (an aminoglycoside acetyltransferase conferring resistance to tobramycin and amikacin), and the non-synonymous mutation T83I on GyrA associated with resistance to fluoroquinolones.
The higher prevalence of ST235 prompted us to look further at this clone. The phylogenetic tree of 49 ST235 isolates comprised two distinct clades with different geographic distribution (Fig. 2C). Clade I $(n=10)$ was represented in five hospitals in the Luzon (north) and Visayas (central) island groups, while clade II $(n=39)$ was represented in 10 hospitals from north to south of the country. The phylogeographic structure of the tree and the relatedness between genomes showed evidence of dissemination of ST235 between hospitals. Within clade Ib (Fig. 2C), one genome from hospital NKI differed from two genomes from hospital BRH by seven and eight SNPs, respectively. Within clade IIb (Fig. 2C), the genetic differences between isolates from the same hospital (mean pairwise SNP differences $36.41 \pm 20.84$, range $0-64$ ) were not significantly different to those between isolates from different hospitals (mean $45.36 \pm 8.12$, range 29-61, Mann-Whitney $\mathrm{U}$ test z-score $=-1.49145, P=0.13622$ ). The close relationships and the common repertoire of resistance genes between isolates from different hospitals support inter-hospital transmission.

The genomes from the hospital VSM $(n=24)$ formed at least three clusters within clade Ilb, two of which exhibited discrete temporal distribution (VSM-2 and VSM-3, Fig. 2C), suggesting that they could represent hospital outbreaks. In agreement with this, the genomes from different patients within clade VSM-3 differed by an average of 11.55 pairwise SNPs (range 0-24). We also identified isolates within VSM-3 that were collected nine or more months apart (Fig. 2C), suggesting that ST235 can either persist in or be reintroduced to the hospital environment.

The distribution of acquired resistance genes and mutations showed that resistance determinants differed between clades I and II, with patterns that were consistent with the acquisition of multiple genes simultaneously by mobile genetic elements. Long-read sequencing of isolate 14ARS-VSM0870, representative of the XDR resistant profile CAZ FEP IPM MEM TZP GEN TOB AMK CIP (marked with an asterisk on Fig. $2 \mathrm{C}$ ), revealed the acquisition of blavim-2, bla oxa-10, catB3, aadA1 (ANT(3")-la) and $\operatorname{acc}\left(6^{\prime}\right)-I b$ within a class 1 integron integrated in the chromosome at position 977774 (Fig. 2D). The ciprofloxacin resistance gene 
Fig. 2. Genomic surveillance of $P$. aeruginosa from the Philippines, 2013-2014
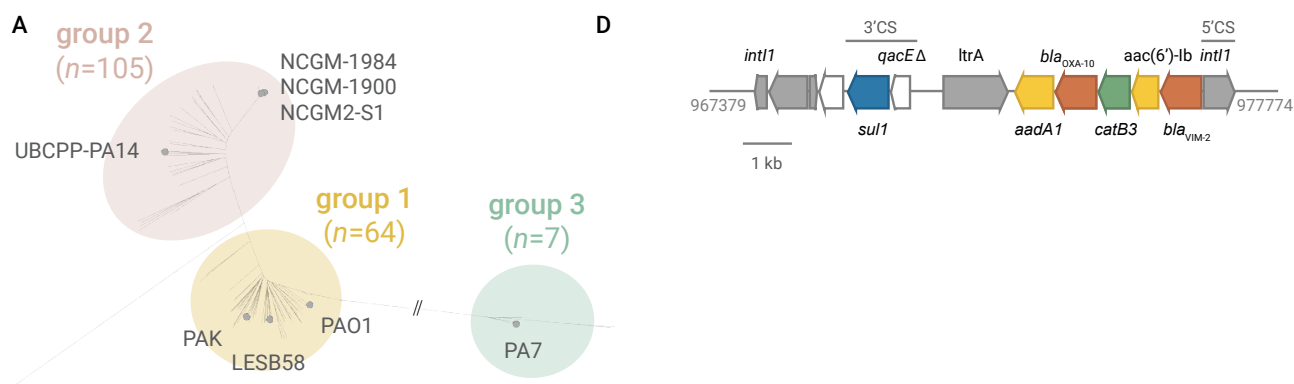

B
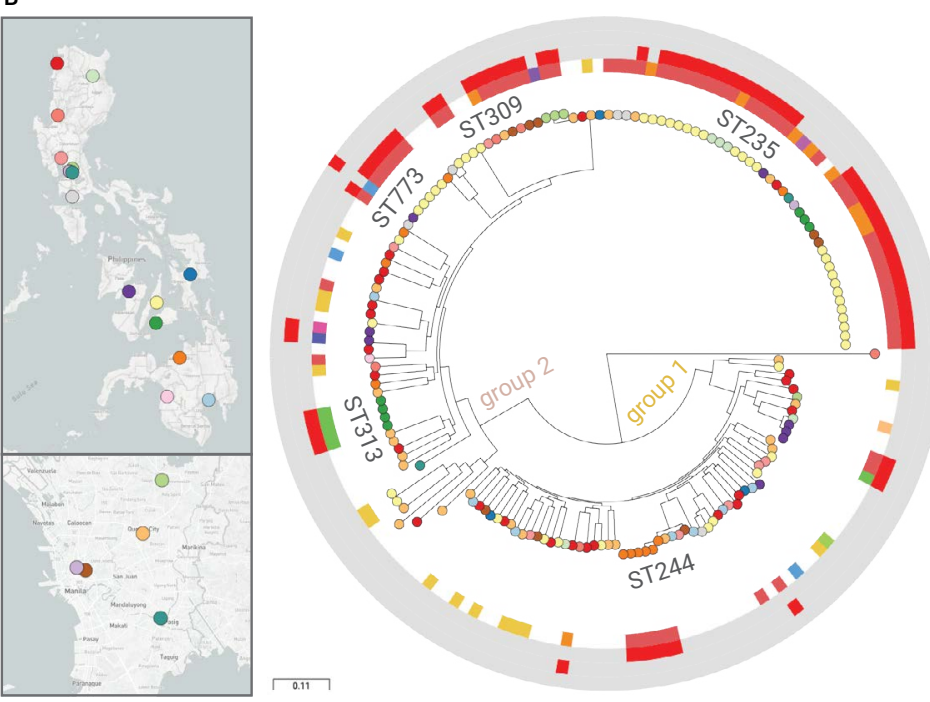

Inner circle: Resistance Profile

$\square$ CAZ FEP IPM MEM GEN TOB

$\square$ CAZ FEP IPM MEM GEN TOB CIP

$\square$ CAZ FEP IPM MEM TZP

$\square$ CAZ FEP IPM MEM TZP GEN TOB AMK

CAZ FEP IPM MEM TZP GEN TOB AMK CIP

$\square$ CAZ FEP IPM MEM TZP GEN TOB CIP

FEP IPM MEM GEN TOB CIP

$\square$ IPM

$\square$ IPM MEM

IPM MEM CIP

$\square$ IPM MEM TZP CIP

$\square$ Susceptible to carbapenems

Outer circles: blaVIM, blaIMP, blaNDM

$\square$ Present

$\square$ Absent

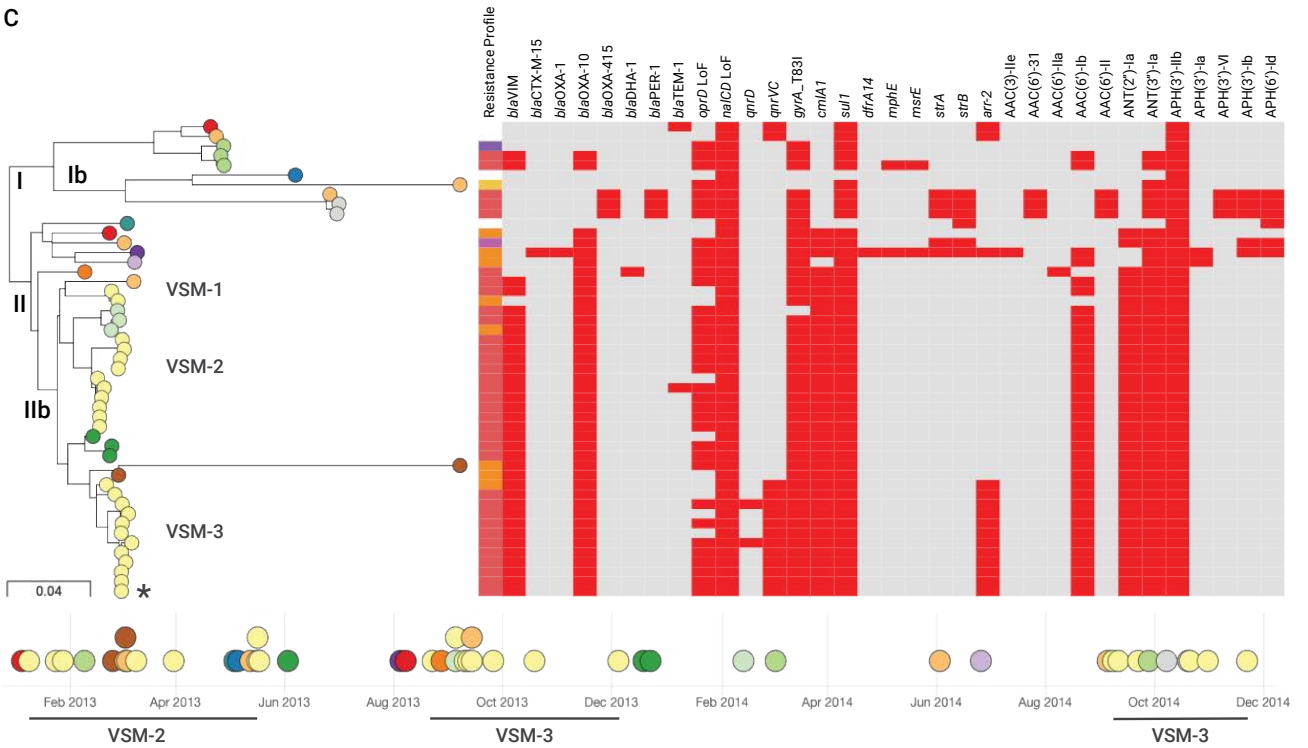

Fig. 2A. Phylogenetic tree of 176 isolates from the Philippines and eight reference genomes, inferred with RAxML from an alignment of 396 194 core SNP sites. The reference genomes are indicated by grey nodes.

Fig. 2B. Phylogenetic tree of 169 isolates from groups 1 and 2 inferred with RAxML from an alignment of 305220 SNP sites obtained after mapping the genomes to the complete genome of strain LESB58 and masking mobile genetic elements from the alignment. The tree leaves are coloured by sentinel site and indicated on the map (left panels, top: Philippines, bottom: detail of the National Capital Region). Tree rings indicate (from inner to outer) the distribution of the carbapenem-resistant profiles and of carbapenemase genes blavim, b/amp and bla and. The data, including the full distribution of resistance determinants, are available at https://microreact.org/project/ARSP_169PAE_2013-2014.

Fig. 2C. Phylogenetic tree of 49 ST235 genomes inferred from an alignment of 1066 SNP sites obtained after mapping the genomes to the complete genome of strain NCGM2-S1 (ST235) and masking mobile genetic elements and recombination regions. The tree leaves are coloured by sentinel site as indicated on the map from Fig. 2B. The tree blocks represent the distribution of the carbapenem-resistant profiles and of acquired resistance genes and mutations. The representative isolate sequenced with long reads is shown with an asterisk. The full data are available at https://microreact.org/project/ARSP_PAE_ST235_2013-14. The scale bars represent the number of SNPs per variable site.

Fig. 2D. Resistance genes acquired en bloc within a class 1 integron in $P$. aeruginosa strain 14ARS-VSM0870. Arrows indicate genes conferring resistance to $\beta$-lactamases (orange), aminoglycosides (yellow), chloramphenicol (green) and sulphonamides (blue), or related to DNA mobilization/integration (grey). 3'CS and 5'CS: conserved segments. 
qnrVC and the rifampin-resistance gene arr-2 were located on a different class 1 integron elsewhere in the genome.

\section{P. aeruginosa from the Philippines in the global context}

We placed the genomes from our retrospective collection in the global context of 904 contemporary $P$. aeruginosa public genomes. This collection of public genomes represented 17 countries and 178 STs, with more than $60 \%$ of the genomes being from Europe ( $n=373$ ) and the United States of America (USA) $(n=205)$. The Philippine genomes were found throughout the tree, indicating that the $P$. aeruginosa population captured in our survey largely represents the global diversity of this pathogen. Notably absent were the epidemic clones ST111 and ST175 (Fig. 3A), which, together with ST235, are responsible for MDR and XDR nosocomial infections worldwide.

A more detailed tree of 96 ST235 genomes of global distribution showed three major clades: clade 1 was represented by isolates from Japan, the Philippines, Poland and Thailand $(n=2)$; clade 2 showed the broadest geographic distribution across four continents and also included isolates from this study $(n=3)$; clade 3 comprised exclusively isolates from the Philippines ( $n=44$, Fig. 3B), which raises the possibility that this lineage of ST235 is characteristic to the Philippines; however, introductions from the other globally dispersed lineages may also occur, as shown in clades 1 and 2.

\section{DISCUSSION}

In this study, we combined WGS and laboratory-based surveillance to characterize susceptible and resistant $P$. aeruginosa circulating in the Philippines in 2013 and 2014, with a particular emphasis on resistance to carbapenems, which increased in the years preceding this survey. Drug-resistant $P$. aeruginosa infections are difficult to treat, resulting in poor patient outcomes. In a tertiary hospital in Manila, severity of illness and mortality rates were significantly higher among patients infected with drug-resistant $P$. aeruginosa than among those infected with susceptible isolates, while median duration of hospital stay was significantly longer. ${ }^{33}$
$P$. aeruginosa strains exhibit a complex interplay between resistance mechanisms, both intrinsic and acquired. ${ }^{34}$ The current gaps in understanding of some of these mechanisms were reflected in the variable concordance between phenotypic and genotypic resistance for the different antibiotics, even for those antibiotics belonging to the same class (aminoglycosides). Our characterization of the carbapenem resistance showed a combination of diverse known mechanisms, from inhibition of antibiotic influx into the cell, to upregulation of antibiotic efflux out of the cell and carbapenemase enzymes. The concordance between phenotypic and genotypic predictions of AMR was high for the carbapenems, but it required a degree of curation of results that is not practical within public health settings.

There are clear limitations in the genomic predictions of AMR for $P$. aeruginosa. First, publicly available, curated databases are not comprehensive of all the known mechanisms. We found no mutations leading to upregulation of the chromosomal cephalosporinase AmpC (b/a $\left.a_{\text {PAO }}\right)$, but an exhaustive search would require additional analyses. Second, the regulatory pathways of some mechanisms are not fully understood, such as those that regulate AmpC. ${ }^{34,35}$ Third, extensive manual curation of some of the predictions is needed to ensure accuracy, for example of the loss-of-function mutations in the oprD gene.

The most prevalent clone in our data set was ST235 (27.8\% of the isolates, $n=49$ ), found throughout the Philippines. ST235 is a well-characterized international epidemic clone causing drug-resistant nosocomial outbreaks. ${ }^{32}$ Isolates carrying $b / a_{\text {VIM-2 }}$ and belonging to ST235 were reported from Malaysia, the Republic of Korea and Thailand. ${ }^{13}$ Using WGS, we showed evidence of potential localized hospital outbreaks of ST235, as well as of persistence or reintroduction of this clone within one hospital. The number of SNP differences between genomes of isolates from different patients (0-24) were consistent with those reported for a persistent outbreak of $P$. aeruginosa in a hospital in the United Kingdom of Great Britain and Northern Ireland. ${ }^{36}$ We also showed evidence of transfer of ST235 between hospitals, with isolates from different hospitals separated by as few as seven SNPs. Patient transfer between hospitals is not common in the Philippines, but the sampling for this 


\section{Fig. 3. $\quad P$. aeruginosa from the Philippines in the global context}

A

ST244

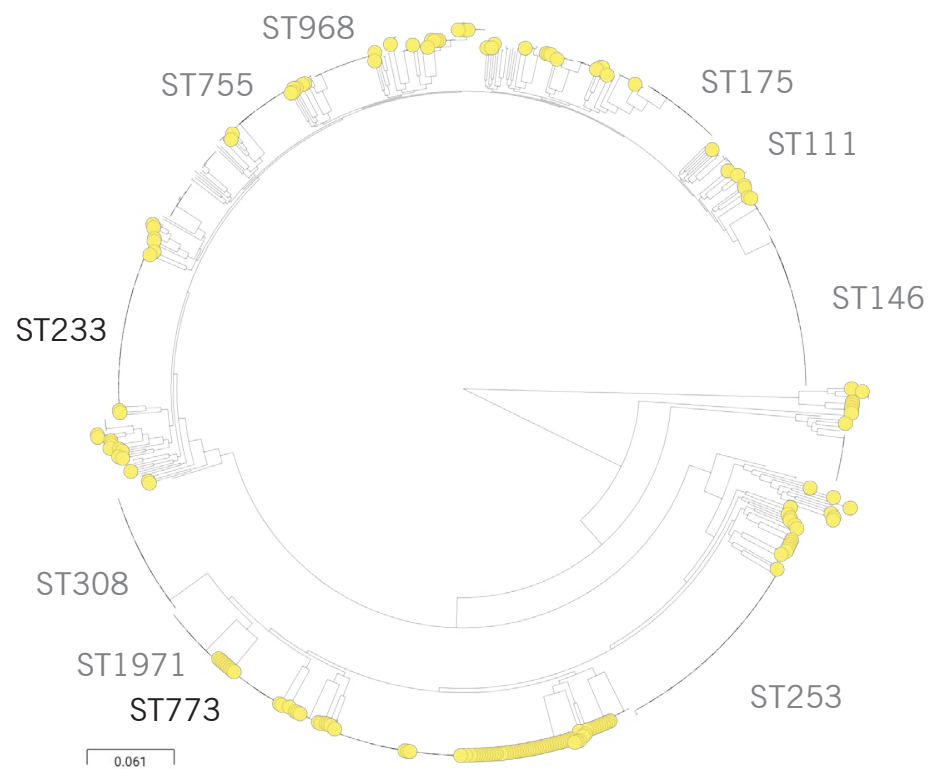

ST235

B
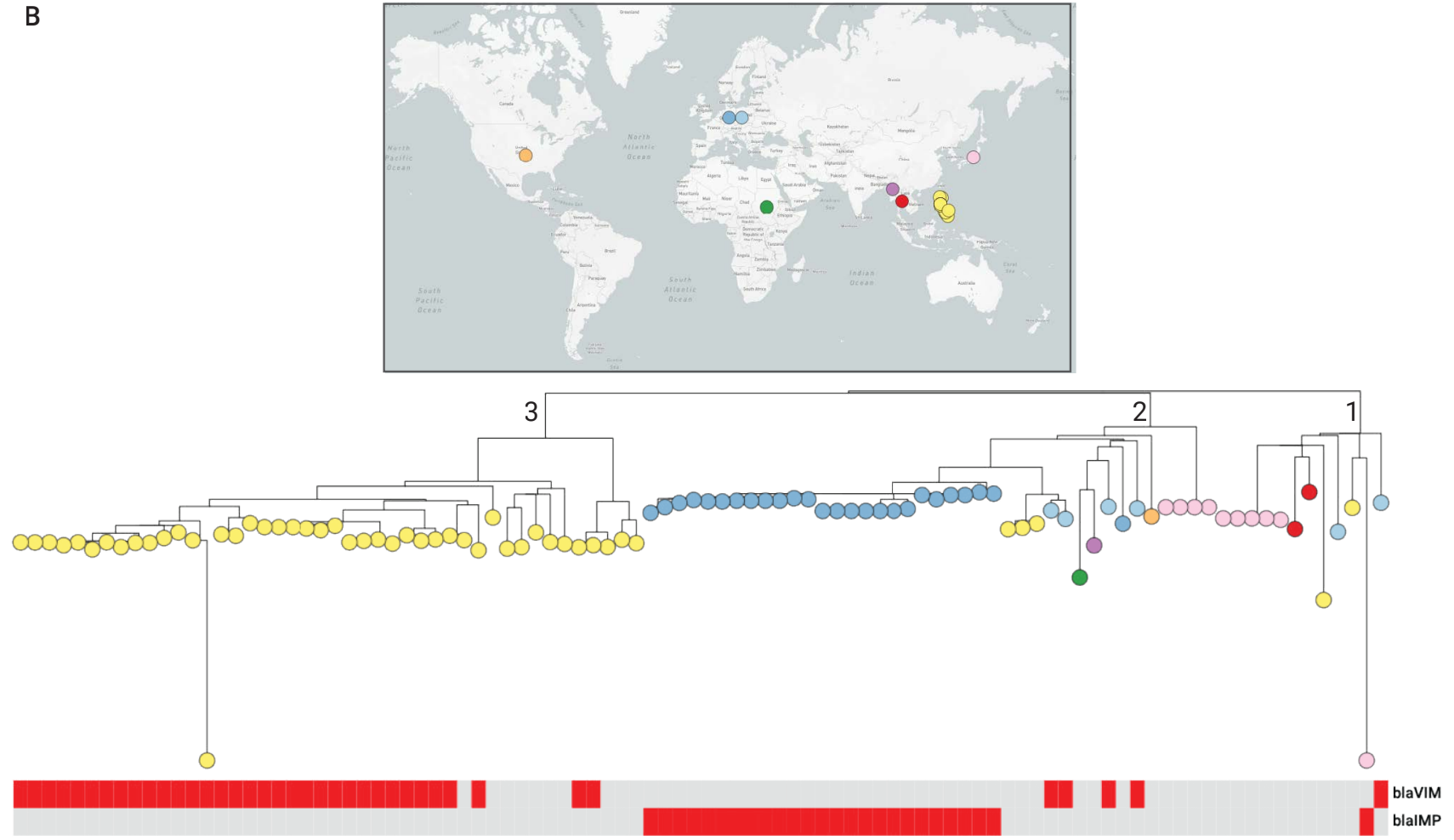

0.03

Fig. 3A. Phylogenetic tree of 904 P. aeruginosa isolates from the Philippines ( $n=176$, this study) and from 57 other countries inferred from 549126 SNP positions. The yellow tree nodes indicate the genomes from this study. The major lineages (STs) are labelled in black if represented by genomes of this study, or in brown if they are not. The data are available at https://microreact.org/project/ARSP_PAE_GLOBAL.

Fig. 3B. Phylogenetic tree of 96 ST235 isolates inferred from an alignment of 1993 SNP positions. The tree leaves are coloured by country as indicated on the map. The tree is annotated with the distribution of blavim and blaımp genes (red: present, grey: absent). The data are available at https://microreact.org/project/ ARSP PAE ST235_GLOBAL. The scale bars represent the number of SNPs per variable site. 
Table 3. Comparison of genomic predictions of antibiotic resistance with laboratory susceptibility testing at the ARSRL

\begin{tabular}{|c|c|c|c|c|c|c|c|}
\hline Antibiotic class & Antibiotic & $\begin{array}{l}\text { Isolates } \\
\text { tested }\end{array}$ & $\begin{array}{l}\text { Resistant } \\
\text { isolates } \\
\text { (AST) }\end{array}$ & $\begin{array}{l}\text { False } \\
\text { positive }\end{array}$ & $\begin{array}{c}\text { False } \\
\text { negative }\end{array}$ & Concordance (\%) & $\begin{array}{l}\text { Acquired resistance } \\
\text { mechanisms }\end{array}$ \\
\hline \multirow[t]{2}{*}{ Carbapenem } & Imipenem & 176 & 100 & 1 & 4 & 97.16 & $b / a_{\text {VIM-2 }}, b / a_{\text {VIM- }}, b / a_{\text {NDM-1 }}$ \\
\hline & Meropenem & 176 & 99 & 2 & 4 & 96.59 & $\begin{array}{l}\text { blaımp-26, OprD loss-of-func- } \\
\text { tion (oprD interrupted, frag- } \\
\text { mented, or missing, presence } \\
\text { of premature STOP, START } \\
\text { codon missing), NalC/D loss- } \\
\text { of-function (nalC missing, } \\
\text { NalC_G71E, S209R, A186T, } \\
\text { NalD_S32N) }\end{array}$ \\
\hline \multirow[t]{3}{*}{ Aminoglycoside } & Gentamicin & 176 & 77 & 0 & 34 & 80.68 & $\begin{array}{l}\text { AAC(3)--Ile, AAC(6')-31, } \\
\text { AAC(6')-Ila, ANT(2")-la }\end{array}$ \\
\hline & Tobramycin & 176 & 78 & 2 & 3 & 97.16 & 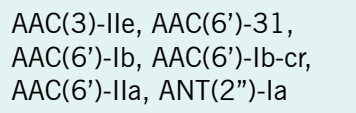 \\
\hline & Amikacin & 176 & 61 & 14 & 4 & 89.77 & $\begin{array}{l}\text { AAC( }\left(6^{\prime}\right)-31, A A C\left(6^{\prime}\right)-I b \\
A A C\left(6^{\prime}\right)-I I a, A P H\left(3^{\prime}\right)-V I\end{array}$ \\
\hline Fluoroquinolone & Ciprofloxacin & 176 & 82 & 5 & 12 & 93.75 & $\begin{array}{l}\text { qnrD, qnrVC, AAC(6')-lb-cr, } \\
\text { GyrA_D87N, D87Y, T83I, } \\
\text { GyrB_E468D, S466F, } \\
\text { ParC_S87L }\end{array}$ \\
\hline
\end{tabular}

AST: antimicrobial susceptibility testing.

study only allows us to hypothesize about a possible role of the community, animals or the environment in the spread of this clone.

It was previously proposed that ST235 emerged in Europe around 1984, coinciding with the introduction of fluoroquinolones, and then disseminated to other regions via two independent lineages, acquiring resistance determinants to aminoglycosides and $\beta$-lactams locally. ${ }^{14}$ Simultaneous acquisition of resistance to multiple antibiotics via integrons, transposons and integrative conjugative elements is well described in $P$. aeruginosa, ${ }^{36}$ and is apparent in the distribution of resistance genes in our genomes. We have shown an example of a class 1 integron carrying six resistance genes in the genetic background of ST235. While this integron shared some features with others previously described in $P$. aeruginosa, ${ }^{13,32}$ such as the $5^{\prime}$ and $3^{\prime}$ conserved segments, ${ }^{37}$ the gene composition and synteny was different, supporting the hypothesis of local acquisition of resistance.

Country-specific ST235 lineages have been reported previously, ${ }^{11,14}$ confirming that country-wide clonal expansions may occur in the context of the global circulation of this clone. A previous longitudinal study showed VIM-2-positive ST235 spreading throughout Belarus, Kazakhstan and the Russian Federation, albeit without the resolution of whole genome data. ${ }^{38}$ The contextualization of our genomes with international ST235 genomes showed a distinct cluster of Philippine genomes with limited genetic variability, suggesting the clonal expansion and geographic dissemination of this lineage across the Philippines. Alternatively, this could be explained by the limited representation of the Western Pacific Region in the collection of global genomes, highlighting the need for public genome data with more even geographical coverage. Our retrospective survey contributed to bridging this gap by making raw sequence data available on public archives.

In conclusion, our detailed description of the epidemiology and resistance mechanisms of ST235 in the Philippines suggests that the burden of XDR $P$. aeruginosa infections in the Philippines may be largely driven by a local lineage of the international epidemic clone ST235. A recent study in a hospital in Jakarta, Indonesia analysed the population composition of $P$. aeruginosa before and after a multifaceted infection control intervention, with the relative abundance of ST235 
Table 4. Distribution of isolates, sequence types (STs), resistance profiles and acquired resistance mechanisms across the 17 sentinel sites ${ }^{a}$

\begin{tabular}{|c|c|c|c|c|c|}
\hline Laboratory & $\begin{array}{l}\text { No. of } \\
\text { Isolates }\end{array}$ & $\begin{array}{l}\text { No. of } \\
\text { STs }\end{array}$ & $\begin{array}{l}\text { Prevalent } \\
\text { ST (no. of } \\
\text { isolates) }\end{array}$ & Resistance profiles & Acquired resistance determinants \\
\hline BGH & 6 & 5 & $309(2)$ & $\begin{array}{c}\text { Susceptible (2) } \\
\text { CAZ FEP IPM MEM TZP GEN TOB CIP (2) } \\
\text { CAZ FEP IPM MEM TZP GEN TOB AMK CIP } \\
\text { (1) } \\
\text { IPM MEM (1) }\end{array}$ & $\begin{array}{c}\text { NaIC/D LOF (2) } \\
\text { IMP-26, AAC(6')-lb, QnrVC1, OprD LOF, NaIC/D LOF (1) } \\
\text { VIM-2, AAC(6')-Ib4, ANT(3")-la, GyrA.D87Y, OprD LOF, } \\
\text { NaIC/D LOF (1) } \\
\text { VIM-2, AAC(6')-Ib4, ANT(3")-Ia, QnrVC1, GyrA.T83I, } \\
\text { OprD LOF, NaIC/D LOF (1) } \\
\text { OprD LOF, NaIC/D LOF (1) }\end{array}$ \\
\hline $\mathrm{BRH}$ & 5 & 3 & $235(2)$ & $\begin{array}{c}\text { CAZ FEP IPM MEM TZP GEN TOB AMK CIP } \\
\text { (3) } \\
\text { Susceptible (2) }\end{array}$ & $\begin{array}{c}\text { AAC(6')-31, AAC(6')-II, ANT(3")-la, APH(3')-VI, GyrA. } \\
\text { T83I, ParC.S87L, OprD LOF, NaIC/D LOF (2) } \\
\text { VIM-2, AAC(6')-Ib4, APH(3')-la, QnrVC1, NaIC/D LOF (1) } \\
\text { NaIC/D LOF (2) }\end{array}$ \\
\hline $\mathrm{CMC}$ & 1 & 1 & 1121 & $\begin{array}{l}\text { CAZ FEP IPM MEM TZP GEN TOB AMK CIP } \\
\text { (1) }\end{array}$ & ANT(2")-la, ANT(3")-la, QnrVC1, NaIC/D LOF (1) \\
\hline CVM & 5 & 3 & $235(3)$ & $\begin{array}{c}\text { CAZ FEP IPM MEM TZP GEN TOB AMK CIP } \\
(2) \\
\text { Susceptible (2) } \\
\text { CAZ FEP IPM MEM TZP GEN TOB CIP (1) }\end{array}$ & $\begin{array}{c}\text { VIM-2, AAC(6')-Ib4, ANT(2")-la, ANT(3")-la, GyrA.T83I, } \\
\text { ParC.S87L, OprD LOF, NaIC/D LOF (2) } \\
\text { NaIC/D LOF (2) } \\
\text { VIM-2, AAC(6')-Ib4, ANT(2")-la, ANT(3")-la, GyrA.T83I, } \\
\text { ParC.S87L, OprD LOF, NaIC/D LOF (1) }\end{array}$ \\
\hline DMC & 7 & 7 & $\begin{array}{l}9,463 \\
381,244 \\
639,303 \\
357(1)\end{array}$ & $\begin{array}{c}\text { Susceptible (3) } \\
\text { CAZ FEP IPM MEM TZP GEN TOB AMK CIP } \\
\text { (2) } \\
\text { IPM MEM (2) }\end{array}$ & $\begin{array}{c}\text { NalC/D LOF (2) } \\
\text { None (1) } \\
\text { ANT(2")-la, ANT(3")-la, APH(3')-VI, QnrVC1, gyrB. } \\
\text { S466F, OprD LOF, NaIC/D LOF (1) } \\
\text { IMP-26, AAC(6')-lb4, ANT(2")-la, ANT(3")-la, } \\
\text { QnrVC1, OprD LOF, NalC/D LOF (1) } \\
\text { OprD LOF, NaIC/D LOF (2) }\end{array}$ \\
\hline EVR & 4 & 4 & $\begin{array}{l}1966 \sim \\
1978,235 \\
1823(1)\end{array}$ & $\begin{array}{c}\text { Susceptible (2) } \\
\text { CIP (1) } \\
\text { CAZ FEP IPM MEM TZP GEN TOB CIP (1) }\end{array}$ & $\begin{array}{c}\text { NalC/D LOF (2) } \\
\text { NalC/D LOF (1) } \\
\text { VIM-2, AAC(6')-Ib4, APH(3')-la, NaIC/D LOF (1) }\end{array}$ \\
\hline FEU & 4 & 2 & $235(3)$ & $\begin{array}{c}\text { CAZ FEP IPM MEM TZP GEN TOB AMK CIP } \\
(2) \\
\text { IPM MEM CIP (1) } \\
\text { Susceptible (1) }\end{array}$ & $\begin{array}{c}\text { VIM-2, AAC(6')-Ib4, ANT(3")-la, GyrA.T83I, ParC.S87L, } \\
\text { OprD LOF, NaIC/D LOF (2) } \\
\text { GyrA.T83I, ParC.S87L, OprD LOF, NaIC/D LOF (1) } \\
\text { NaIC/D LOF (1) }\end{array}$ \\
\hline GMH & 8 & 3 & $313(4)$ & $\begin{array}{c}\text { CAZ FEP IPM MEM TZP GEN TOB AMK (4) } \\
\text { CAZ FEP IPM MEM TZP GEN TOB AMK CIP } \\
(4)\end{array}$ & $\begin{array}{c}\text { VIM-2, AAC(6')-Ib4, NaIC/D LOF (4) } \\
\text { VIM-2, AAC(6')-Ib4, ANT(2")-la, ANT(3")-la, GyrA.T83I, } \\
\text { ParC.S87L, OprD LOF, NaIC/D LOF (2) } \\
\text { VIM-2, AAC(6')-Ib4, ANT(2")-la, ANT(3")-la, GyrA.T83I, } \\
\text { ParC.S87L, NaIC/D LOF (1) } \\
\text { VIM-6, AAC(6')-Ib4, GyrA.T83I, ParC.S87L, NaIC/D LOF } \\
\text { (1) }\end{array}$ \\
\hline JLM & 7 & 7 & $\begin{array}{l}244,1597 \\
381,261,2 \\
330,309 \\
316(1)\end{array}$ & $\begin{array}{l}\text { Susceptible (6) } \\
\text { FEP TZP TOB CIP (1) }\end{array}$ & $\begin{array}{c}\text { NaIC/D LOF (5) } \\
\text { None (1) } \\
\text { AAC(6')-Ib-cr, GyrA.T83I, ParC.S87L, NaIC/D LOF (1) }\end{array}$ \\
\hline MAR & 24 & 20 & 357 (3) & $\begin{array}{c}\text { Susceptible (14) } \\
\text { IPM MEM (6) } \\
\text { CAZ FEP IPM MEM TZP (2) } \\
\text { CAZ FEP IPM MEM TZP GEN TOB CIP (1) } \\
\text { GEN TOB CIP (1) }\end{array}$ & $\begin{array}{c}\text { NalC/D LOF (14) } \\
\text { OprD LOF, NaIC/D LOF (4) } \\
\text { AAC(6')-lb, OprD LOF, NaIC/D LOF (1) } \\
\text { gyrB.E468D, OprD LOF, NalC/D LOF (1) } \\
\text { OprD LOF, NaIC/D LOF (2) } \\
\text { ANT(2")-la, ANT(3")-la, GyrA.T83I, ParC.S87L, NaIC/D } \\
\text { LOF (1) } \\
\text { QnrVC1, NaIC/D LOF (1) }\end{array}$ \\
\hline $\mathrm{MMH}$ & 8 & 5 & $272 \sim(3)$ & $\begin{array}{l}\text { CAZ FEP IPM MEM TZP GEN TOB AMK CIP } \\
\text { (2) } \\
\text { CAZ FEP IPM MEM GEN TOB (1) } \\
\text { CAZ FEP IPM MEM GEN TOB CIP (1) } \\
\text { CAZ FEP IPM MEM TZP (1) } \\
\text { CAZ FEP IPM MEM TZP GEN TOB AMK (1) } \\
\text { CAZ FEP IPM MEM TZP GEN TOB CIP (1) } \\
\text { IPM MEM TZP CIP (1) }\end{array}$ & $\begin{array}{r}\text { VIM-2, AAC(3)-Ile, APH(3')-la, APH(3')-VI, OprD LOF, } \\
\text { NaIC/D LOF (2) } \\
\text { IMP-26, AAC(6')-lb4, APH(3')-la, APH(3')-VI, NaIC/D } \\
\text { LOF (1) } \\
\text { IMP-26, AAC(6')-Ib4, APH(3')-la, APH(3')-VI, NaIC/D } \\
\text { LOF (1) } \\
\text { NDM-1, ANT(3")-la, APH(3')-VI, OprD LOF, NaIC/D LOF } \\
(1) \\
\text { VIM-2, AAC(3)-lie, APH(3')-la, APH(3')-VI, NaIC/D LOF } \\
(1) \\
\text { AAC(3)-Ile, AAC(6')-lb4, ANT(3")-la, APH(3')-la, GyrA. } \\
\text { T83I, ParC.S87L, OprD LOF, NaIC/D LOF (1) } \\
\text { gyrB.E468D, OprD LOF, NaIC/D LOF (1) }\end{array}$ \\
\hline
\end{tabular}




\begin{tabular}{|c|c|c|c|c|c|}
\hline Laboratory & $\begin{array}{l}\text { No. of } \\
\text { Isolates }\end{array}$ & $\begin{array}{l}\text { No. of } \\
\text { STs }\end{array}$ & $\begin{array}{l}\text { Prevalent } \\
\text { ST (no. of } \\
\text { isolates) }\end{array}$ & Resistance profiles & Acquired resistance determinants \\
\hline NKI & 26 & 21 & $235(5)$ & $\begin{array}{c}\text { Susceptible (15) } \\
\text { CAZ FEP IPM MEM TZP GEN TOB AMK CIP } \\
\text { (3) } \\
\text { CIP (3) } \\
\text { CAZ FEP GEN TOB AMK CIP (1) } \\
\text { CAZ FEP TZP (1) } \\
\text { FEP IPM MEM GEN TOB CIP (1) } \\
\text { IPM (1) } \\
\text { IPM MEM (1) }\end{array}$ & $\begin{array}{c}\text { NalC/D LOF (14) } \\
\text { None (1) } \\
\text { AAC(6')-31, AAC(6')-II, ANT(3")-la, APH(3')-VI, GyrA. } \\
\text { T83I, ParC.S87L, OprD LOF, NalC/D LOF (1) } \\
\text { VIM-2, AAC(6')-Ib4, ANT(2")-la, ANT(3")-la, GyrA.T83I, } \\
\text { VIM-2, AAC(6')-Ib4, ANT(3")-la, QnrVC1, NalC/D LOF (1) } \\
\text { GyrA.D87N, OprD LOF, NalC/D LOF (1) } \\
\text { NalC/D LOF (1) } \\
\text { QnrVC1, NaIC/D LOF (1) } \\
\text { GyrA.T83I, ParC.S87L, NalC/D LOF (1) } \\
\text { NalC/D LOF (1) } \\
\text { ANT(2")-la, ANT(3")-la, GyrA.T83I, ParC.S87L, OprD } \\
\text { LOF, NalC/D LOF (1) } \\
\text { OprD LOF, NalC/D LOF (1) } \\
\text { ANT(3")-la, OprD LOF, NaIC/D LOF (1) }\end{array}$ \\
\hline NMC & 11 & 6 & $244(6)$ & $\begin{array}{l}\text { CAZ FEP IPM MEM TZP GEN TOB AMK CIP } \\
\text { (6) }\end{array}$ & $\begin{array}{l}\text { VIM-2, AAC(6')-lb4, ANT(3")-la, GyrA.T83I, ParC.S87L, } \\
\text { OprD LOF, NalC/D LOF (4) } \\
\text { AAC(6')-lia, ANT(2")-la, ANT(3")-la, GyrA.T83I, ParC. } \\
\text { S87L, OprD LOF, NaIC/D LOF (1) } \\
\text { VIM-2, AAC(6')-lb4, QnrVC1, GyrA.T83I, ParC.S87L, } \\
\text { OprD LOF, NaIC/D LOF (1) } \\
\text { NalC/D LOF (4) } \\
\text { None (1) }\end{array}$ \\
\hline RMC & 2 & 2 & $\begin{array}{l}1632,235 \\
\text { (1) }\end{array}$ & $\begin{array}{c}\text { CIP (1) } \\
\text { Susceptible (1) }\end{array}$ & $\begin{array}{l}\text { GyrA.T83I, ParC.S87L, NaIC/D LOF (1) } \\
\text { NalC/D LOF (1) }\end{array}$ \\
\hline SLH & 1 & 1 & 235 & CAZ FEP IPM MEM TZP GEN TOB CIP (1) & $\begin{array}{c}\text { AAC(6')-Ib4, ANT(3")-la, APH(3')-la, GyrA.T83I, ParC. } \\
\text { S87L, OprD LOF, NaIC/D LOF (1) }\end{array}$ \\
\hline STU & 9 & 6 & $309(3)$ & $\begin{array}{c}\text { CAZ FEP IPM MEM TZP GEN TOB AMK CIP } \\
\text { (3) } \\
\text { IPM MEM (3) } \\
\text { CAZ FEP IPM MEM TZP GEN TOB CIP (2) }\end{array}$ & $\begin{array}{c}\text { VIM-2, AAC(6')-Ib4, ANT(3")-Ia, QnrVC1, GyrA.T83I, } \\
\text { OprD LOF, NaIC/D LOF (3) } \\
\text { OprD LOF, NaIC/D LOF (2) } \\
\text { NalC/D LOF (1) } \\
\text { VIM-2, AAC(6')-Ib4, ANT(2")-la, ANT(3")-la, GyrA.T83I, } \\
\text { ParC.S87L, NaIC/D LOF (1) } \\
\text { VIM-2, AAC(6')-Ib4, ANT(2")-la, ANT(3")-la, GyrA.T83I, } \\
\text { ParC.S87L, OprD LOF, NaIC/D LOF (1) } \\
\text { None (1) }\end{array}$ \\
\hline VSM & 48 & 16 & $235(24)$ & $\begin{array}{c}\text { Susceptible (12) } \\
\text { IPM MEM (3) } \\
\text { CAZ FEP IPM MEM TZP GEN TOB CIP (2) } \\
\text { CAZ (1) }\end{array}$ & 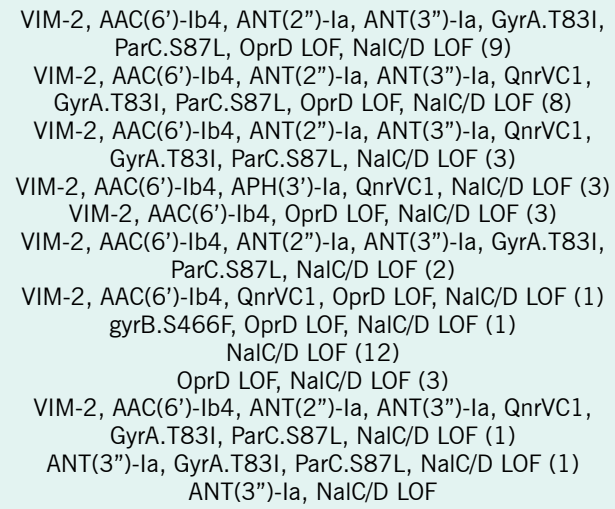 \\
\hline
\end{tabular}

a Only genes and mutations associated with the antibiotic classes tested are shown ( $\beta$-lactamases, aminoglycosides, and fluoroquinolones). The full complement can be found in https://microreact.org/project/ARSP_176PAE_2013-2014.

LOF: loss-of-function.

almost halved in the 10 months post-intervention. ${ }^{39}$ This highlights the importance of hospital infection control and of preventive measures to contain the spread of this high-risk clone.

\section{Acknowledgements}

None.

\section{Funding}

This work was supported by a Newton Fund award from the Medical Research Council (United Kingdom) MR/N019296/1 and the Philippine Council for Health Research and Development. Additional support was provided by the National Institute for Health Research (United Kingdom) Global Health Research Unit on 
Genomic Surveillance of AMR (16/136/111) and by a research grant U01CA207167 from the National Institutes of Health (USA).

\section{Conflicts of Interest}

The authors declare no conflicts of interest.

\section{References}

1. Rossolini GM, Mantengoli E. Treatment and control of severe infections caused by multiresistant Pseudomonas aeruginosa. Clin Microbiol Infect. 2005 Jul;11 Suppl 4:17-32. doi:10.1111/j.14690691.2005.01161.x pmid:15953020

2. Chawla R. Epidemiology, etiology, and diagnosis of hospitalacquired pneumonia and ventilator-associated pneumonia in Asian countries. Am J Infect Control. 2008 May;36(4) Suppl:S93-100. doi:10.1016/j.ajic.2007.05.011 pmid:18468551

3. Navoa-Ng JA, Berba R, Arreza Galapia Y, Rosenthal VD, Villanueva VD, Tolentino MCV, et al. Device-associated infections rates in adult, pediatric, and neonatal intensive care units of hospitals in the Philippines: International Nosocomial Infection Control Consortium (INICC) findings. Am J Infect Control. 2011;39(7):548-54.

4. López-Causapé C, Cabot G, Del Barrio-Tofiño E, Oliver A. The versatile mutational resistome of Pseudomonas aeruginosa. Front Microbiol. 201804 6;9:685. doi:10.3389/fmicb.2018.00685 pmid:29681898

5. Mitchell KF, Safdar N, Abad CL. Evaluating carbapenem restriction practices at a private hospital in Manila, Philippines as a strategy for antimicrobial stewardship. Arch Public Health. 2019 Jul 4;77(1):31. doi:10.1186/s13690-019-0358-9 pmid:31312447

6. Antimicrobial Resistance Surveillance Program 2013 annual report. Manila: Antimicrobial Resistance Surveillance Reference Laboratory; 2014. Available from: https://arsp.com.ph/publications, accessed 1 March 2020.

7. Antimicrobial Resistance Surveillance Program 2014 annual report. Manila: Antimicrobial Resistance Surveillance Reference Laboratory; 2015. Available from: https://arsp.com.ph/publications, accessed 1 March 2020.

8. Antimicrobial Resistance Surveillance Program 2018 annual report. Manila: Antimicrobial Resistance Surveillance Reference Laboratory; 2019. Available from: https://arsp.com.ph/publications, accessed 1 March 2020.

9. Viedma E, Juan C, Acosta J, Zamorano L, Otero JR, Sanz F, et al. Nosocomial spread of colistin-only-sensitive sequence type 235 Pseudomonas aeruginosa isolates producing the extendedspectrum beta-lactamases GES- 1 and GES- 5 in Spain. Antimicrob Agents Chemother. 2009 Nov;53(11):4930-3. doi:10.1128/ AAC.00900-09 pmid:19738007

10. Wi YM, Choi JY, Lee JY, Kang Cl, Chung DR, Peck KR, et al. Emergence of colistin resistance in Pseudomonas aeruginosa ST235 clone in South Korea. Int J Antimicrob Agents. 2017 Jun;49(6):767-9. doi:10.1016/j.jjantimicag.2017.01.023 pmid:28392440
11. Miyoshi-Akiyama T, Tada T, Ohmagari N, Viet Hung N, Tharavichitkul P, Pokhrel BM, et al. Emergence and spread of epidemic multidrug-resistant Pseudomonas aeruginosa. Genome Biol Evol. 2017 Dec 1;9(12):3238-45. doi:10.1093/gbe/evx243 pmid:29202180

12. Castanheira M, Bell JM, Turnidge JD, Mendes RE, Jones RN. Dissemination and genetic context analysis of bla(VIM-6) among Pseudomonas aeruginosa isolates in Asian-Pacific Nations. Clin Microbiol Infect. 2010 Feb;16(2):186-9. doi:10.1111/j.1469_ 0691.2009.02903.x pmid:19673963

13. Kim MJ, Bae IK, Jeong SH, Kim SH, Song JH, Choi JY, et al. Dissemination of metallo- $\beta$-lactamase-producing Pseudomonas aeruginosa of sequence type 235 in Asian countries. J Antimicrob Chemother. 2013 Dec;68(12):2820-4. doi:10.1093/jac/dkt269 pmid:23843299

14. Treepong P, Kos VN, Guyeux C, Blanc DS, Bertrand X, Valot B, et al. Global emergence of the widespread Pseudomonas aeruginosa ST235 clone. Clin Microbiol Infect. 2018 Mar;24(3):258-66. doi:10.1016/j.cmi.2017.06.018 pmid:28648860

15. Juayang AC, Lim JPT, Bonifacio AFV, Lambot AVL, Millan SM, Sevilla V, et al. Five-year antimicrobial susceptibility of Pseudomonas aeruginosa from a local tertiary hospital in Bacolod City, Philippines. Trop Med Infect Dis. 2017;2(3): 28. doi:10.3390\%2 Ftropicalmed2030028 pmid: 30270886

16. Litzow JM, Gill CJ, Mantaring JB, Fox MP, MacLeod WB, Mendoza $\mathrm{M}$, et al. High frequency of multidrug-resistant gram-negative rods in 2 neonatal intensive care units in the Philippines. Infect Control Hosp Epidemiol. 2009 Jun;30(6):543-9. doi:10.1086/597512 pmid:19435448

17. Quick J, Cumley N, Wearn CM, Niebel M, Constantinidou C, Thomas CM, et al. Seeking the source of Pseudomonas aeruginosa infections in a recently opened hospital: an observational study using whole-genome sequencing. BMJ Open. 2014 Nov 4;4(11):e006278. doi:10.1136/bmjopen-2014-006278 pmid:25371418

18. Argimón S, Masim MAL, Gayeta JM, Lagrada ML, Macaranas PKV, Cohen V, et al. Integrating whole-genome sequencing within the National Antimicrobial Resistance Surveillance Program in the Philippines. Nat Commun. 2020 Jun 1;11(1):2719. doi:10.1038/ s41467-020-16322-5 pmid:32483195

19. Global antimicrobial resistance surveillance system (GLASS) report: early implementation 2016-2017. Geneva: World Health Organization; 2017.

20. M100S Performance standards for antimicrobial susceptibility testing. 26th ed. Pennsylvania: Clinical and Laboratory Standards Institute; 2016.

21. Magiorakos AP, Srinivasan A, Carey RB, Carmeli Y, Falagas ME, Giske $C G$, et al. Multidrug-resistant, extensively drug-resistant and pandrug-resistant bacteria: an international expert proposal for interim standard definitions for acquired resistance. Clin $\mathrm{Mi}-$ crobiol Infect. 2012 Mar;18(3):268-81. doi:10.1111/j.14690691.2011.03570.x pmid:21793988

22. Hunt M, Mather AE, Sánchez-Busó L, Page AJ, Parkhill J, Keane JA, et al. ARIBA: rapid antimicrobial resistance genotyping directly from sequencing reads. Microb Genom. 2017 Sep 4;3(10):e000131. doi:10.1099/mgen.0.000131 pmid:29177089 
23. Jolley KA, Maiden MC. BIGSdb: Scalable analysis of bacteria genome variation at the population level. BMC Bioinformatics 2010 Dec 10;11(1):595. doi:10.1186/1471-2105-11-595 pmid:21143983

24. Cury J, Jové T, Touchon M, Néron B, Rocha EP. Identification and analysis of integrons and cassette arrays in bacterial genomes. Nucleic Acids Res. 2016 Jun 2;44(10):4539-50. doi:10.1093/ nar/gkw319 pmid:27130947

25. Croucher NJ, Page AJ, Connor TR, Delaney AJ, Keane JA, Bentley $\mathrm{SD}$, et al. Rapid phylogenetic analysis of large samples of recombinant bacterial whole genome sequences using Gubbins. Nucleic Acids Res. 2015 Feb 18;43(3):e15. doi:10.1093/nar/gku1196 pmid:25414349

26. Page AJ, Taylor B, Delaney AJ, Soares J, Seemann T, Keane JA, et al. SNP-sites: rapid efficient extraction of SNPs from multiFASTA alignments. Microb Genom. 2016 Apr 29;2(4):e000056. doi:10.1099/mgen.0.000056 pmid:28348851

27. Stamatakis A. RAxML version 8: a tool for phylogenetic analysis and post-analysis of large phylogenies. Bioinformatics. 2014 May 1;30(9):1312-3. doi:10.1093/bioinformatics/btu033 pmid:24451623

28. Price MN, Dehal PS, Arkin AP. FastTree 2-approximately maximum-likelihood trees for large alignments. PLoS One. 2010 Mar 10;5(3):e9490. doi:10.1371/journal.pone.0009490 pmid:20224823

29. David S, Reuter S, Harris SR, Glasner C, Feltwell T, Argimon S, et al.; EuSCAPE Working Group; ESGEM Study Group. Epidemic of carbapenem-resistant Klebsiella pneumoniae in Europe is driven by nosocomial spread. Nat Microbiol. 2019 Nov;4(11):1919-29. doi:10.1038/s41564-019-0492-8 pmid:31358985

30. McArthur AG, Waglechner N, Nizam F, Yan A, Azad MA, Baylay AJ, et al. The comprehensive antibiotic resistance database. Antimicrob Agents Chemother. 2013 Jul;57(7):3348-57. doi:10.1128/ AAC.00419-13 pmid:23650175

31. Freschi L, Jeukens J, Kukavica-Ibrulj I, Boyle B, Dupont MJ, Laroche $\mathrm{J}$, et al. Clinical utilization of genomics data produced by the international Pseudomonas aeruginosa consortium. Front Microbiol. 2015 Sep 29;6:1036. doi:10.3389/fmicb.2015.01036 pmid:26483767
32. Oliver A, Mulet X, López-Causapé C, Juan C. The increasing threat of Pseudomonas aeruginosa high-risk clones. Drug Resist Updat. 2015 Jul-Aug;21-22:41-59. doi:10.1016/j.drup.2015.08.002 pmid:26304792

33. Dimatatac EL, Alejandria MM, Montalban C, Pineda C, Ang C, Delino R. Clinical outcomes and costs of care of antibiotic resistant Pseudomonas aeruginosa Infections. Philipp J Microbiol Infect Dis. 2003;31(4):159-67.

34. Lister PD, Wolter DJ, Hanson ND. Antibacterial-resistant Pseudomonas aeruginosa: clinical impact and complex regulation of chromosomally encoded resistance mechanisms. Clin Microbiol Rev. 2009 Oct;22(4):582-610. doi:10.1128/CMR.00040-09 pmid:19822890

35. Juan C, Torrens G, González-Nicolau M, Oliver A. Diversity and regulation of intrinsic $\beta$-lactamases from non-fermenting and other Gram-negative opportunistic pathogens. FEMS Microbio Rev. 2017 Nov 1;41(6):781-815. doi:10.1093/femsre/fux043 pmid:29029112

36. Snyder LA, Loman NJ, Faraj LA, Levi K, Weinstock G, Boswell $\mathrm{TC}$, et al. Epidemiological investigation of Pseudomonas aeruginosa isolates from a six-year-long hospital outbreak using high-throughput whole genome sequencing. Euro Surveill. 2013 Oct 17;18(42):20611. doi:10.2807/1560-7917. ES2013.18.42.20611 pmid:24176582

37. Kung VL, Ozer EA, Hauser AR. The accessory genome of Pseudomonas aeruginosa. Microbiol Mol Biol Rev. 2010 Dec;74(4):62141. doi:10.1128/MMBR.00027-10 pmid:21119020

38. Edelstein MV, Skleenova EN, Shevchenko OV, D'souza JW, Tapalski DV, Azizov IS, et al. Spread of extensively resistant VIM-2-positive ST235 Pseudomonas aeruginosa in Belarus, Kazakhstan, and Russia: a longitudinal epidemiological and clinical study. Lancet Infect Dis. 2013 Oct;13(10):867-76. doi:10.1016/ S1473-3099(13)70168-3 pmid:23845533

39. Pelegrin AC, Saharman YR, Griffon A, Palmieri M, Mirande $C$, Karuniawati $A$, et al. High-risk international clones of carbapenem-nonsusceptible Pseudomonas aeruginosa endemic to Indonesian intensive care units: impact of a multifaceted infection control intervention analyzed at the genomic level. MBio. 2019 Nov 12;10(6):e02384-19. doi:10.1128/mBio.02384-19 pmid:31719179 